\title{
Research on the Status Quo of English Majors' Group Presentation in Private Colleges
}

\author{
Zhou Wei ${ }^{1}$ \\ ${ }^{1}$ Foreign Language Department, Guangzhou Huashang College, Guangzhou, Guangdong, China \\ 254626619@qq.com
}

\begin{abstract}
This study aims to explore the status quo of group presentations for English majors in private colleges. An online questionnaire was conducted on 294 college English majors and 12 of them took part in semi-structured interviews. Both quantitative and qualitative analyses were conducted and revealed that there were advantages and problems in the present group presentation in private colleges' classes. Results of the study indicate that: 1) English teachers in private colleges generally like to implement the teaching method of group presentation. English students in private colleges generally believe that it is necessary to implement group presentation in English teaching, but the degree of their like of group presentation is at a medium level;2) students are generally satisfied with the presentation environment except for the classroom layout; 3 ) teachers can arrange group size reasonably, leave enough time for students to prepare and give students help and feedback in class, but they are somewhat deficient in topic arrangement and after-class tutoring; 4) the presenters' English proficiency and presentation skills need to be improved. These findings were discussed and implications were suggested on language teaching.
\end{abstract}

Keywords: group presentation, environment, teachers, presenters

\section{民办高校英语专业学生小组合作课堂展示现状的调查 研究}

周 薇 1

1 广州华商学院外国语学院, 广州, 广东, 中国 254626619@qq.com

\section{摘要}

本研究考查了民办高校英语专业学生小组合作课堂展示的现状。通过对 294 名英专学生的问卷调查和 12 位同 学的半结构化访谈的定量和定性分析发现, 目前民办高校英专学生课堂小组展示存在优点, 但也存在问题。具 体表现为：1）民办高校英语老师普遍喜欢实施课堂小组展示这种教学方式, 民办高校英专学生普遍认为在英 语教学中有必要开展小组展示, 但是对这种教学方式的喜爱程度不高；2）除了课堂布局以外, 学生普遍对展 示环境表示满意；3）授课教师在合理安排小组人数、留足够时间给学生做准备和在课堂上给到学生帮助和反 馈方面做得较好, 但在话题安排和课后辅导这两方面稍显欠缺；4）展示者的英语水平和展示技巧还需提升。 这些发现对外语教学能提供些许启示。

关键词: 课堂小组展示; 环境; 教师; 展示者

\section{1. 引言}

随着任务型教学理念的兴起, 越来越多的高校教 师和中学教师要求学生在语言课堂里做 presentation (陈述, 展示, 报告), 相关的研究也 同时进行。课堂展示指的是学生个人或小组在课堂里 就某个话题或书中的某些知识点向全班讲解的教学 实践活动 ${ }^{[1]}$ below。课堂展示包含个人展示和小组展 示。目前关于课堂展示的研究涉及到以下几个方面: 小组合作课堂展示在大学英语教学中的实施情况以 及它对提高学生英语水平、增强学习兴趣和建立良好
的师生关系的作用 ${ }^{[2]}$ ，教师为提高小组课堂展示效果 所做的行动研究 ${ }^{[3]}$, 教师对学生课堂展示的有效指导 ${ }^{[4]}$, presentation 环节的多模态教学设计 ${ }^{[5]}$, 小组展 示前准备阶段的同伴对话 ${ }^{[6]}$ 等。

纵观前人研究, 关于 “小组合作课堂展示” 的研 究主要针对公办院校的研究生 ${ }^{[7]}$, 公办院校的非英语 专业本科生 ${ }^{[6]}$, 公办院校的英语专业本科生 ${ }^{[9]}$ 和高职 院校英语专业学生 ${ }^{[10]}$, 而民办学校英语专业学生却得 不到应有的关注。由于教学对象的语言水平和课程要 求存在巨大差异，同样是实施课堂小组展示，公办院 校学生和民办高校学生对此法的感受未必相同。因此, 
有必要深入调查民办高校英语专业学生对此种教学 模式的理解和感受, 以利于深入了解课程要求、学习 者特点和教师课堂教学三者之间的关系。具体而言, 本研究将以广东某民办高校的英语专业学生为调查 对象, 利用问卷和访谈来收集数据, 并尝试回答以下 研究问题:

1）小组合作课堂展示在民办高校英语专业课堂 实施情况如何?

2）民办高校英语专业学生对小组合作课堂展示 的态度是怎样的?

3) 目前的小组合作课堂展示有哪些优点和不足? 如何改善这些不足?

\section{2. 理论框架}

小组合作课堂展示主要体现基于以下几个理论:

\section{(1) 小组合作学习}

20 世纪 70 时代, 合作学习在美国兴起。美国学 者约翰逊兄弟俩认为合作学习就是在教学上使学生 通过小组形式共同活动, 以此来促进学生自己和同伴 的学习 ${ }^{[11]}$ 。合作学习理论着眼于学生与同伴之间的互 动。在小组合作展示中, 从话题的选定, 资源的共享, 进一步的交流到最后的成果展示, 无时不体现着学生 与同伴的互动。在互动过程中, 学生不但能够培养团 队精神, 而且也能实现优势互补, 提高学习效率, 进 而个体也得到了发展。

(2) 输入输出理论

Krashen ${ }^{[12]}$ 的输入理论告诉我们, 外语学习者需 要大量的可理解性输入。输入固然重要, Swain ${ }^{[13]}$ 的输 出理论也强调了源源不断的可理解性的输出对于外 语学习者的重要性。在课堂小组展示教学中, 学生的
输入主要来自两个方面, 一个是在准备任务时所查阅 的相关资料以及与同伴的交流过程中，另一个是在课 堂中, 学生不仅仅接受到来自教师的语言输入, 同伴 的展示也为他们提供了丰富的语言输入。同时, 对于 展示者而言, 要输出可理解性的语言, 他必然会注意 自身语言表达的准确性, 这样可以进一步促进他意识 到语言形式和所要表达的意义之间的相互关系。同时, 输出活动能够促进展示者认识到自身存在的语言问 题和困难, 以及对目标语领域内未知或知之不足的内 容的重视。

\section{（3）任务型学习理论}

任务型学习是基于杜威等人的体验式 “做中学” 教育哲学思想而提出的教学法, 其核心理念是通过模 拟现实生活中语言运用的活动, 让语言学习者在完成 任务的过程中习得语言 ${ }^{[14]}$ 。任务型学习最核心的概念 是任务。关于什么是任务, Ellis s $^{[15]}$ 提出了四条判断标 准: 1) 聚焦于意义的表达; 2) 交际双方存在某种信 息差；3）学习者主要依靠自己的资源（语言和非语 言）来完成任务；4）有明确的、非聚焦语言的结果。 任务型语言教学的理念在课堂小组展示教学中得到 了体现。学生通过与小组成员协商确定任务后, 积极 利用各种语言的和非语言的资源来完成任务, 并且整 理撰写报告, 即有一个明确的结果。在展示之前, 在 某个特定话题方面，展示者和听众之间存在着信息差， 展示者要自信流利地把自己的观点表达出来并和听 众互动, 必然聚焦于意义。

\section{3. 研究设计}

\section{1 研究对象}

通过便利抽样和标准抽样相结合, 本研究选取广 东某民办高校 294 名英专学生作为问卷调查对象。参 与调查的学生个人信息如表所示。

表 1 学生个人信息表

\begin{tabular}{ccccccccc}
\hline 性别 & 频数 & 百分比 & 就读年级 & 频数 & 百分比 & $\begin{array}{c}\text { 是 否 当 众 } \\
\text { 频数 }\end{array}$ & 百分比 \\
\hline 男 & 32 & 10.92 & 大一 & 91 & 30.95 & 是 & 261 & 88.78 \\
女 & 262 & 89.12 & 大二 & 62 & 21.09 & 否 & 33 & 11.22 \\
& & & 大三 & 112 & 38.1 & & & \\
\hline
\end{tabular}

\section{2. 研究工具}

\subsection{1. 问卷调查}

本研究在参考蔡静静 ${ }^{[8]}$ 的调查问卷的基础上, 做 了些许修改。该问卷由两个部分组成。第一部分收集 受试的个人信息, 包括性别、就读年级和是否当场做 过课堂展示。第二部分询问受试对小组合作课堂展示
的看法, 包括 24 道单选题。该 24 道单选题又可以分 为四个维度: 第 1-5 题是关于学生对课堂小组展示的 态度（详见表 2)，第 6-9 题是关于受试对课堂展示 环境的看法（详见表 3)，第 10-15 题是关于受试对 实施课堂展示教学法的授课教师的看法 (详见表 4), 第 16-24 题是受试对展示者和受试自己的看法 (详见 表 5）。笔者对几位受试进行了试测, 然后根据试测 的结果对问卷的个别表述进行了修正。 
笔者通过探索性因子分析和验证性因子分析检 验了问卷的结构效度。用 SPSS 26.0 对数据进行探索 性因子分析结果显示：KMO 值 $=.929>$. 8, 巴特 莱特球形检验值 $=3848.151(\mathrm{df}=276, \mathrm{p}=.000$ $<$.05)，方差贡献率达到 $60.835 \%$ 。通过用 $\operatorname{Amos} 22.0$ 对问卷结构进行验证性因子分析，发现 Chi-square /df、CFI、IFI、TLI、GFI 和 RMSEA 等 几项指数显示模型与数据达到较好的拟合, 说明问卷 具有较好的结构效度。问卷整体信度系数 $\alpha=.932$, 各下属因子的信度系数分别为: 态度因子. 878 , 环境 因子. 802 , 教师因子. 828 , 学生因子. 895 , 说明该问 卷具有较好的内部一致性。

\subsection{2. 半结构化访谈}

从受试中随机抽取 12 名学生进行访谈。访谈内 容与问卷调查内容基本一致, 目的是为了得到相互佐 证的回答。笔者对访谈内容采用一对一的方式进行录 音。

\section{3. 数据收集}

问卷调查于 2021 年 3 月展开。300 份问卷主要 通过电子邮件发放, 回收了 294 份, 有效问卷的回收 率为 $98 \%$ 。访谈也于 2021 年 3 月开展。研究者随机抽 取了 12 名学生, 根据研究目的自行编著了半结构式 访谈提纲。访谈所要考察的内容如下：（1）受访者所 在班级是否有实施课堂小组合作展示。问题有：你的 任课老师有让你们做课堂小组展示吗? 如果有, 频率 是多高?（2）受访者对课堂小组合作展示的态度。问 题有: 你喜欢课堂小组合作展示吗? 参加的积极性高 吗?（3）目前课堂小组合作展示实施情况的优缺点 及改进措施。问题有: 你认为目前课堂小组合作展示 实施情况如何? 有哪些优点? 哪些缺点? 有何建议 让它实施得更好呢? 你可以从课堂环境、教师和学习 者三个方面来谈。

\section{4. 数据分析}

问卷调查采用李克特五级量表, 单选题的给分方 式是：完全不同意 (1)、不同意 (2)、不确定 (3)、 同意 (4) 和完全同意 (5)。其中括号里的数字是分 值。笔者对问卷数据进行了描述性统计分析, 并对访 谈得到的数据进行转写与分析。

\section{4. 结果分析与讨论}

\section{1. 课堂小组展示在民办高校英语专业中的 实施情况}

问卷调查的结果显示, 参加问卷的 294 名受试中 有 261 名当众做过课堂小组展示, 比率为 $89 \%$ 。参加 访谈的很多受试都表明 90\%的任课老师都会让他们做 小组课堂展示。这说明课堂小组展示这种教学方法在
民办高校英专课堂中得到了普遍实施。任课教师意识 到了这种教学法的好处, 喜欢施行这种教学法。

\section{2. 对课堂小组展示的态度}

这方面内容体现在问卷的第 1-5 题。表 2 归纳了 受试对这部分问题回答的频数及百分比分布。关于民 办高校英专学生对课堂小组展示的态度，从表 2 可以 看出, $49 \%$ 受试喜欢课堂小组展示, 但是他们认为在 英语教学中有必要开展课堂小组展示 (61.44\%)，小 组展示能增强学习英语积极性 $(60.75 \%)$ ，能够增强 自信和团队合作意识 $(65.18 \%)$ ，能够提高学生运用 多媒体的能力 $(74.41 \%)$ ，这些结果与周杏莉 ${ }^{[2]}$ 的研 究结果部分一致, 但是与她的发现 “ $76.7 \%$ 的同学表 示喜欢并继续小组合作课堂展示的活动形式” 不一致, 这令人反思。首先, “以学生为中心” 的教学理念在 课堂小组展示中得到了体现。在访谈中, 一位大二的 同学说 “相对于 '老师讲, 我们听” 这种被动的学习 方法, 我更加喜欢和同学一起探究问题后在课堂上自 信地展示自我。”很多同学都认为课堂小组展示可以 丰富知识, 增强学习动机。正如李小平 ${ }^{[16]}$ 等研究证明: 培养学生对学习的投入意愿和情感, 变 “被动学习” 为“主动学习”至关重要。其次, 根据二语习得理论, 外语学习者需要输入和输出双管齐下。课堂小组展示 可以为展示者提供更多的输出机会，同时也为听众提 供了更多的输入机会。正如一位大一的同学所说: “以 前高中学英语就是不断的做题, 讲题, 考试, 觉得英 语学习毫无趣味可言。到了大学, 老师在课堂采用小 组展示这种教学方式, 我终于迎来了用英语自由表达 自我的机会, 所以我很喜欢这样的教学方式。”还有 一位同学说: “我的英语和电脑能力都较弱, 但是我 愿意向能力强的同学请教, 同学们也乐意帮助我, 这 让我觉得学习不再是负担。”虽然课堂小组展示有如 上优点, 但是还不到一半的同学喜欢它, 这说明目前 这种教学方式在实施过程中还存在不少问题。

\section{3. 目前课堂小组展示的优点和缺点}

本研究的研究问题 3 是关于目前民办高校英专 学生课堂小组展示实施情况的优点和缺点的, 这部分 的内容体现在受试对问卷第 6-24 题的回答。表 3 表 明受试对展示环境的看法。表 4 表明受试对任课教师 的看法。表 5 表明受试对展示者和受试自身的看法。

表 3 表明, 63.14\%的受试认为当前的课堂学生人 数有利于课堂小组展示, $55.29 \%$ 的受试认为当前的课 堂布局有利于课堂小组展示, $65.53 \%$ 的受试认为当前 的课堂硬件设备有利于课堂小组展示, 69. $97 \%$ 的受试 认为他们能够从学校图书馆和互联网上查阅到做展 示时所需要的资料。这些数据表明, 大多数受试对当 前的展示环境看法积极, 主要原因可能是英语专业学 生都是小班教学, 这一点不同于至少 50 个人的大学 英语课堂。在这些数据里, 受试对当前课堂布局的认 可率比其他项的认可率要低一些。有一个大四的同学 
在受访过程中说: “课室里的麦克风固定在讲台上, 教室里有一些多余的桌椅未搬走, 这些妨碍了我跟同 学们互动。”

表 4 中的问题 10-15 是关于在课堂小组展示中, 受试对任课教师的做法的看法。受试对问题 11 和 14 赞同最多 (69.38\%)，问题 15 次之（赞同 64.62\%, 不赞同 $5.1 \%$ )，接着是问题 13 (赞同 $61.9 \%$, 不赞同 $7.82 \%)$ ，赞同问题 10 的最少 (28.23\%)，且不确定 的达到 58. 16\%; 赞同问题 12 的也比较少 (53.74\%), 不确定的有 $36.05 \%$ 。这些结果表明, 授课教师在 “给 学生充分时间准备” 和 “给展示者即时反馈” 这两方 面实施较好, 对 “在课堂上及时帮助学生课堂展示” 和 “合理分配小组成员人数” 两方面实施相对较弱。 在 “课外指导学生小组展示” 方面实施较弱, 在 “分 配学生感兴趣的话题” 实施最弱。任课教师课外指导 学生小组展示少, 可能是三个原因造成的: 第一, 任 课老师太相信学生能力, 希望学生依靠小组的力量解 决问题; 第二, 任课老师除了上课以外, 还有其他事 情要忙, 比如说科研和家庭。第三, 学生害怕麻烦老 师, 不主动联系老师。比如在采访中, 有一位大四学 生说: “我的老师除了上课外, 还要照顾两个孩子, 所以我都不好意思去打扰她。再说了, 如果我去找老 师求助的话, 会显得我解决问题的能力不强。”此外, 受试对教师分配的话题不太满意可能是因为教师为 了自己方便, 没有与学生商定展示话题, 这也反映出 不少教师未将“以学生为中心”的教学理念真正落实。 有一位大二同学说 “老师给我们分配的话题不够生活
化, 我感觉不太实用。”

民办院校英专学生对展示者和自身的看法体现 在问卷的第 16-24 题的回答中。表 5 显示, 受试赞同 比例由高到低依次为问题 16(67\%)、问题 24(66.32\%)、 问题 17(62.92\%)、问题 20(51.7\%)、问题 21(43.53\%)、 问题 23(36.39\%)、问题 18(35.37\%)、问题 22(36.39\%), 问题 19 (31.63\%)。这些结果表明，大多数被试对展 示者的展示情况不满意, 展示者提高展示水平的空间 还很大。具体解释可从展示者和听众两个方面入手: 第一, 有些展示者在展示前没有做好充分的准备再加 上自身英语水平较弱，不标准的发音导致听众对展示 内容失去了兴趣。第二, 展示者缺乏展示技巧。他们 在 PPT 上面打了很多文字, 有些展示者纯粹就是照着 PPT 念, 内容多, 翻页快, 不顾及听众的反应, 没法 做到言简意赅和与听众互动。这两个因素导致听众对 展示者展示内容兴趣不大。第三, 听众怀疑展示内容 的权威性, 毕竟展示者不是老师。第四, 听众怕情感 上伤害展示者进而影响同学关系, 故而没有给到展示 者足够的反馈。在采访过程中, 有位大一的同学说: “有的同学在展示的时候声音小, 发音不标准, 我都 不想听她说了。”有位大三同学说: “整个展示过程 就是展示者的个人秀, 他就是把 PPT 上的文字读完就 完事了。”有位大二同学说 “我还是喜欢听老师讲, 这样至少保证了内容的可信度。”有位大四同学说“除 非展示者亲自问我们或者老师叫我们进行点评，我们 一般是不会主动说出展示者的缺点的, 这样容易伤害 同学感情。

表 2: 民办院校英专学生对课堂小组展示的态度：频数和百分比（ $\mathrm{N}=294$, 括号里为 \%

\begin{tabular}{lccccl}
\hline \multicolumn{1}{c}{ 问卷题目 } & 完全同意 & 同意 & \multicolumn{1}{c}{ 不确定 } & 不同意 & 完全不同意 \\
\hline $\begin{array}{l}\text { 1.我喜欢课堂小组展示。 } \\
\text { 2. 英语教学中有必要开展 }\end{array}$ & $29(9.78)$ & $127(43.2)$ & $105(35.71)$ & $39(13.27)$ & $6(2.04)$ \\
课堂小组展示。 & $152(51.7)$ & $87(29.59)$ & $21(7.14)$ & $5(1.7)$ \\
$\begin{array}{l}\text { 3. 小组展示能增强我学习 } \\
\text { 英语积极性。 }\end{array}$ & $29(9.86)$ & $150(51.02)$ & $76(25.85)$ & $29(9.86)$ & $10(3.4)$ \\
$\begin{array}{l}\text { 4. 小组展示能增强我的自 } \\
\text { 信和团队合作意识。 }\end{array}$ & $33(11.2)$ & $159(54.08)$ & $78(26.53)$ & $18(6.12)$ & $6(2.04)$ \\
$\begin{array}{l}\text { 5.小组展示能提高我运用多 } \\
\text { 媒体的能力。 }\end{array}$ & $36(12.24)$ & $183(62.24)$ & $57(19.39)$ & $13(4.42)$ & $5(1.7)$ \\
\hline
\end{tabular}

表 3：民办院校英专学生对课堂展示环境的看法：频数和百分比（ $\mathrm{N}=294 ，$ 括号里为\%）

\begin{tabular}{lccccc}
\hline \multicolumn{1}{c}{ 问卷题目 } & 完全同意 & 同意 & 不确定 & 不同意 & 完全不同意 \\
\hline $\begin{array}{l}\text { 6.当前的课堂学生人数有利于 } \\
\text { 小组展示。 }\end{array}$ & $26(8.84)$ & $160(54.42)$ & $86(29.25)$ & $20(6.8)$ & $2(0.68)$ \\
$\begin{array}{l}\text { 7.当前的课堂布局有利于小组 } \\
\text { 展示。 }\end{array}$ & $18(6.12)$ & $144(48.98)$ & $97(32.99)$ & $32(10.88)$ & $3(1.02)$ \\
$\begin{array}{l}\text { 8.当前的课堂硬件设备有利于 } \\
\text { 小组展示。 }\end{array}$ & $23(7.82)$ & $169(57.48)$ & $79(26.87)$ & $19(6.46)$ & $4(1.36)$ \\
$\begin{array}{l}\text { 9. 我能从图书馆和互联网查 } \\
\text { 阅到展示所需资料。 }\end{array}$ & $21(7.14)$ & $185(62.93)$ & $72(24.49)$ & $12(4.08)$ & $4(1.36)$ \\
\hline
\end{tabular}

表 4: 民办院校英专学生对任课教师的看法：频数和百分比（ $\mathrm{N}=294 ，$ 括号里为\%）

\begin{tabular}{lccccc}
\hline \multicolumn{1}{c}{ 问卷题目 } & 完全同意 & 同意 & 不确定 & 不同意 & 完全不同意 \\
\hline $\begin{array}{l}\text { 10. 我喜欢老师分配给我的话 } \\
\text { 题。 }\end{array}$ & $8(2.72)$ & $75(25.51)$ & 171（58.16） & 35(11.9) & $5(1.7)$ \\
\hline
\end{tabular}




\begin{tabular}{|c|c|c|c|c|c|}
\hline $\begin{array}{l}\text { 11. 老师留给我们充足的时间准 } \\
\text { 备展示材料。 }\end{array}$ & $25(8.5)$ & $179(60.88)$ & $80(27.21)$ & $9(3.06)$ & $1(0.34)$ \\
\hline $\begin{array}{l}\text { 12. 在学生准备课堂展示的过 } \\
\text { 程中, 老师提供了足够的帮助与 } \\
\text { 指导。 }\end{array}$ & $12(4.08)$ & $146(49.66)$ & $106(36.05)$ & $27(9.18)$ & $3(1.02)$ \\
\hline $\begin{array}{l}\text { 13. 在学生进行课堂展示的过 } \\
\text { 程中, 老师提供了足够的帮助与 } \\
\text { 指导。 }\end{array}$ & $16(5.44)$ & 166 (56.46) & $89(30.27)$ & $20(6.8)$ & $3(1.02)$ \\
\hline $\begin{array}{l}\text { 14. 展示结束后, 我从老师那里 } \\
\text { 得到了足够的反馈。 }\end{array}$ & $24(8.16)$ & $180(61.22)$ & $75(25.51)$ & $14(4.76)$ & $1(0.34)$ \\
\hline $\begin{array}{l}\text { 15. 当前的课堂展示小组成员 } \\
\text { 人数适中。 }\end{array}$ & $20(6.8)$ & $170(57.82)$ & $89(30.27)$ & $9(3.06)$ & $6(2.04)$ \\
\hline
\end{tabular}

表 5: 民办院校英专学生对展示者和自身的看法：频数和百分比（ $\mathrm{N}=294$, 括号里为\%）

\begin{tabular}{|c|c|c|c|c|c|}
\hline 问卷题目 & 完全同意 & 同意 & 不确定 & 不同意 & 完全不同意 \\
\hline $\begin{array}{l}\text { 16. 在准备过程中, 我们小组成 } \\
\text { 员经常交流意见。 }\end{array}$ & $22(7.48)$ & $175(59.52)$ & $70(23.81)$ & $18(6.12)$ & $9(3.06)$ \\
\hline $\begin{array}{l}\text { 17. 课堂展示过程中, 展示者的 } \\
\text { 音量适中。 }\end{array}$ & $14(4.76)$ & $171(58.16)$ & $94(31.97)$ & $14(4.76)$ & $1(0.34)$ \\
\hline $\begin{array}{l}\text { 18. 课堂展示过程中, 展示者的 } \\
\text { 英语发音标准。 }\end{array}$ & $9(3.06)$ & $95(32.31)$ & $156(53.06)$ & $32(10.88)$ & $2(0.68)$ \\
\hline $\begin{array}{l}\text { 19. 课堂展示过程中, 展示者与 } \\
\text { 听众积极互动。 }\end{array}$ & $15(5.1)$ & $78(26.53)$ & $132(44.9)$ & $60(20.41)$ & $9(3.06)$ \\
\hline $\begin{array}{l}\text { 20. 展示者所展示内容具有条理 } \\
\text { 性, 易于理解。 }\end{array}$ & $17(5.78)$ & $135(45.92)$ & $127(43.2)$ & $13(4.42)$ & $2(0.68)$ \\
\hline 21．展示者做展示时言简意赅。 & $18(6.12)$ & $110(37.41)$ & $136(46.26)$ & $27(9.18)$ & $3(1.02)$ \\
\hline $\begin{array}{l}\text { 22.其他同学对展示者所展示内 } \\
\text { 容兴趣很大。 }\end{array}$ & $16(5.44)$ & $78(26.53)$ & $156(53.06)$ & $38(12.93)$ & $6(2.04)$ \\
\hline $\begin{array}{l}\text { 23. 我做完展示后, 从同学那里 } \\
\text { 得到了足够的反馈。 }\end{array}$ & $11(3.74)$ & $96(32.65)$ & $124(42.18)$ & $55(18.71)$ & $8(2.72)$ \\
\hline $\begin{array}{l}\text { 24. 展示结束后, 我会总结和反 } \\
\text { 思, 以使自己进步。 }\end{array}$ & $32(10.88)$ & $163(55.44)$ & $76(25.85)$ & $20(6.8)$ & $3(1.02)$ \\
\hline
\end{tabular}

\section{5. 结论}

本研究调查民办院校英专学生对英语课堂小组 展示的态度和实施情况。发现如下:

第一, 课堂小组展示在英专课堂得到了普遍实施。 学生普遍认同英语课堂小组展示能增强学生学习英 语积极性, 增强自信和团队合作意识, 提高运用多媒 体的能力, 也认为在英语教学中有必要开展课堂小组 展示, 但是对课堂小组展示的喜爱程度不到 $50 \%$ 。

第二, 目前的英语课堂小组展示实施中, 学生普 遍对当前的课堂人数和硬件条件感到满意, 授课教师 能够留给学生充足的准备时间, 合理分配小组成员人 数, 在课堂上及时给与学生指导和反馈。多数学生乐 意与同伴交流意见解决问题, 并且在展示结束后会总 结反思, 争取进步。

第三, 目前的英语课堂小组展示中也存在一定的 问题。具体表现为：（1）为了提高展示的效果, 当前 的课堂布局还有待完善。（2）教师在选择展示话题 时, 未真正做到 “以学生为中心” , 在课后也没有及 时给到学生帮助和指导。（3）展示者的展示水平不
高, 与听众互动效果差。这些发现对外语教学的启示 是:

首先, 课室管理者应鼓励教师和学生说出自己的 需求, 按需改善课堂布局, 如在教室内安装移动话筒, 教务处在排课时应为英专学生安排较为空旷的教室, 这样有利于课堂互动。

其次, 授课教师需要将 “以学生为中心” 落地实 施。课堂小组展示的话题不应该只由教师决定, 学生 有一定的自主选择权, 最终话题的确定应该是师生双 方协商的结果 ${ }^{[4]}$ 。教师应该兼顾课程主题和学生的需 求和爱好。除了课堂指导外, 授课教师应鼓励学生课 后 “不懂就问”，及时解决问题。教师也应在课后多 跟学生情感联系, 提高学习效果。

再次, 学生应该提高自身英语水平和课堂展示技 巧。老师也有必要对学生进行课堂展示的技巧的培训。 展示者展示水平高, 自然容易吸引听众。此外, 听众 也需要尊重展示者的努力, 认真聆听并即时给予反馈。

\section{REFERENCES}

[1] Zhang, L.H. (2004) The Enlightenment of Presentation in American Classroom. Journal of 
Liaoning Teachers College(Social Sciences Edition), 3: $38-48$.

[2] Zhou, X.L. (2010) Group Presentation in College English Teaching: Practice and Reflections. Journal of Zhejing Normal University(Social Sciences), 35: 108-112.

[3] Li, X.Q., Zhang, H., Liu, D. (2013) On the Approaches to Enhancing the Effect of Class Presentation in Group Work. Shangdong Foreign Language Teaching Journal, 34: 62-67.

[4] Wang, A.Y. (2011) Students' Classroom Presentation and Teachers' Effective Guidance. Journal of Education Exploration, 6: 77-78.

[5] Yang, Y., Bai, S., Yuan, J.Y. (2015) A Constructive Study on Initiative in Presentation of College English Teaching under the Context of "Threemulti". Journal of Technology Enhanced Foreign Languages, 3: 24-29.

[6] Han, L. (2020). A Study of Peer Dialogue During Group Presentation Preparations in College English Classes Based on Sociocultural Theory. (Unpublished master's dissertation).Shandong Normal University, Shandong, China.

[7] Yang, B. (2006) The Role of Students' Classroom Presentation in Postgraduate English Teaching. Journal of Foreign Language Teaching, 3: 47-49.

[8] Cai, J. J.(2015). Research on the Status Quo of English Postgraduates' Class Presentation. (Unpublished master's dissertation). Central China
Normal University, Hubei, China.

[9] Zhang, Y.M., Li, T. (2019) A Study on the Teaching Effects of Group Presentation in Classroom on English Majors. Journal of Hubei Open Vocational College, 32: 186-187.

[10] He, Y. (2012) Application of Classroom Presentation Method to Improve Vocational Ability of Students Majoring in Foreign Trade. Journal of Education and Occupation, 24: 158-160.

[11] Wang, T. (2001) Cooperative Learning: Principles and Strategies. Xueyuan Press, Beijing.

[12] Krashen, S.D. (1985) The Input Hypotheses: Issue and Implications. New York: Pergamon Press, Oxford.

[13] Swain, M. (1995). "Three functions of output in second language learning". In G. Cook \& B. Seidlhofer (eds.). Principles and Practice in Applied Linguistics. Oxford University Press, Oxford.

[14] Xie, Y.H., Chen, Q.L. (2019) Exploring College EFL Teachers' Perceptions and Implementation of Taskbased Language Teaching. Journal of Modern Foreign Languages, 42: 267-279.

[15] Ellis, R. (2009). Task-based language teaching: Sorting out the misunderstandings. International Journal of Applied Linguistics 19: 221-246.

[16] Li, X.P., Guo, J.L. (2005) Study on the Correlativity of Students' Learning Attitude and Behavior. Journal of Studies of Psychology and Behavior, 3: 265-267. 